\title{
Analysis of FLOSS Communities as Learning Contexts
}

\author{
Sara Fernandes ${ }^{1,2(\bowtie)}$, Antonio Cerone ${ }^{1}$, and Luis Soares Barbosa ${ }^{2}$ \\ ${ }^{1}$ United Nations University - International Institute for Software Technology, \\ Macao SAR, China \\ \{sara.fernandes, antonio\}@iist.unu.edu \\ 2 HASLab/INESC TEC, University of Minho, Braga, Portugal \\ 1sb@di.uminho.pt
}

\begin{abstract}
It can be argued that participating in Free/Libre Open Source Software (FLOSS) projects can have a positive effect in the contributor's learning process. The need to collaborate with other contributors and to contribute to a project can motivate and implicitly foster learning. In order to validate such statements, it is necessary to (1) study the interactions between FLOSS projects' participants, and (2) explore the didactical value of participating in FLOSS projects, designing an appropriate questionnaire asking FLOSS contributors about their experience in FLOSS projects. In this paper, we illustrate how this questionnaire was designed and disseminated. We conclude the paper with results from 27 FLOSS projects contributors, determining that, not only they contribute and collaborate to the project and its community, but also that FLOSS contributors see that this type of activity can be regarded as a complement to formal education.
\end{abstract}

Keywords: FLOSS $\cdot$ Communities of practice $\cdot$ Learning awareness

\section{Introduction}

Within 15 years the Web has grown from a group work tool for scientists at CERN ${ }^{1}$ into a global information space with more than a billion users [1]. Currently, it is both maintaining its roots as a read/write tool and also entering a new, more social and participatory phase. In particular, it is becoming a participatory tool for people to learn and share knowledge. These trends lead to the feeling that the Web is entering a "second phase" - a new and improved version, also defined as Web 2.0, which is more than a set of new technologies and services. It is an enabler for participation and interactions between users and used as an educative tool. However, there is a significant debate over the alleged advantages and disadvantages of incorporating new technologies into mainstream education; particularly, to foster competence development of students [2]. To address such concerns, new pedagogical and information design perspectives emerge and are closely coupled with Web 2.0 philosophy. Authors

\footnotetext{
${ }^{1}$ Conseil Européen pour la Recherche Nucléaire (European Council for Nuclear Research).
} 
like Chatti, Jarke and Frosch-Wilke [3], present the concept of social software as a tool for augmenting human social and collaborative abilities; Downes [4], promotes the creation of an individual learning network using simple social tools; Happ [5], brings Web 2.0 into the mainstream advocating that is it more than technology and the human-element; Sclater [6], presents the pros and cons of using social networking systems such as Facebook in Learning Management Systems; or Wilson et al. [7] that explores a new design pattern for personal learning environments.

These perspectives help to rethink the purpose of technology-enhanced learning environments in education, to question the existing industry standards and to open the way towards competence development of learners.

According to Stephen Downes [6], knowledge informs learning; the learning outcomes inform community; and the community in turn creates knowledge. Looking from a reverse perspective, knowledge builds community, while community defines what is learned, and what is learned becomes knowledge. These three aspects community, learning and knowledge, essentially model the same phenomenon, representations of communications and structures that are created by individuals interacting and exchanging experiences (knowledge) within communities [8].

As an example of the learning process explained above, Free/Libre Open Source Software (FLOSS) communities consist of heterogeneous groups of independent volunteers, who interact among them driven by different motivations [9]. Software developed by these communities is driven by collaborative, social modes of interaction and knowledge exchange, providing an example of peer-production [10].

Our research work focuses on studying how contributors to FLOSS projects learn and whether and how they recognize the learning process they experience through their contributions. Within such research frame, Fernandes, S. et al. [11] reported an initial experience using a stratified sampling, considering few FLOSS contributors playing different roles and with different demographics - i.e. gender and age. This paper extends such work by presenting results of a more extended sample with 27 respondents, committed to different activities, with diverse gender and age, but also representing different countries and possessing different backgrounds. In particular, the aim of this paper is to (1) study the interactions between FLOSS projects participants, and (2) assess the didactical value of their communities. The main contribution of the results presented here is bringing better understanding of how FLOSS contributors interact and the didactical value of such activity. The rest of this paper is structured as follows. Section 2 introduces some background and related work. Section 3 presents the methodology and the description of the instrument used for data collection. Section 4 presents results, while Sect. 5 the analysis of the results. Finally, Sect. 6 presents some conclusions pointing to envisaged future work.

\section{Background and Related Work}

At the end of 2006 Time magazine's Person of the Year was "You" [12]. On the cover of the magazine, and underneath the title of the award, is a picture of a Personal Computer (PC) with a mirror in the place of the screen, reflecting not only the face of the user, but also the general feeling that 2006 was the year of the Web - a new 
improved, user-generated web. This award and recent trends have led to a feeling that the Web is more and more a source of knowledge and that the activities performed there can be seen as a mean to develop skills.

As an example of collaborative and participatory trends, we focus on FLOSS projects. FLOSS projects are developed using the Web and it has been accepted that the participation in such projects represent a potential positive impact on the contributor's learning processes. The FLOSS community itself provides a valuable, though partial, source of information [10].

In fact, the use of FLOSS projects as learning tools have already gained significant supporters in higher education institutions, and implemented in regular courses. Brocco and Frapolli [13] report on the use of FLOSS projects in a computer science class at the University of Fribourg. Lundell et al. [16] report their experience from a practical assignment at the University of Skövde in Sweden. Papadopoulos et al. [14] reports on the use of an instructional method that utilizes FLOSS projects as tools for teaching software engineering at the Department of Informatics at the University of Thessaloniki in Greece.

In our previous work, we presented preliminary results obtained by conducting a stratified sampling for collecting data to study the learning process of contributors to a FLOSS project [11]. This paper extends such work, focusing on the FLOSS community and its contributors. In [11], and as a way to analyze whether FLOSS has a positive effect on contributors' learning, we decided to run an online questionnaire targeting few FLOSS contributors. The results, although with no statistical relevance, served to pave the way to further broader investigations. This paper extends the analysis mentioned above, after a re-design of the questionnaire and its dissemination to a broader audience.

Our hypothesis remains the same: long-term participation in FLOSS projects can have a positive effect in the contributor's learning process. And our main goal with the revised questionnaire continues to be to understand of how FLOSS projects contributors learn and whether and how contributors recognize the learning process them experience.

\section{Methodology}

This section provides details about the data collection process; in particular, the questionnaire construction and design, as well as the dissemination process.

\subsection{Questionnaire Design}

The questionnaire was structured into three main sections: Section A aims at collecting respondents' demographics; Section B aims at collecting data about the respondent's interaction with the project community, the motivations to start and continue contributing to FLOSS project; and Section C surveys where the respondents exploit the potential of FLOSS projects as learning environments. Each section comprises different types of questions. The questionnaire was formulated using 
open-ended and closed-ended questions. In the open-ended questions the possible responses were not given, allowing the respondent to write down the answers in his/ her own words. In the closed-ended questions, possible answers were provided for the respondent to tick the category that best describes his or her choice. In such questions, the clause "Other/please explain" was included to accommodate any response not listed. The use of these two forms of questions revert to the fact that close-ended questions are extremely useful for eliciting factual information and open-ended questions for seeking opinions, attitudes and perceptions.

In the closed-ended questions, we not only allowed multi-selection answers but also provided three types of Likert Scale answers: (1) to analyze the respondent's perception, including values like Strongly disagree, Disagree, Not sure/Not applicable, Agree, and Strongly agree; and (2) to analyze the frequency of certain respondent's behavior, including values like Ever, Once every year, Once a month, At least 3 times per month, and More than 3 times per month; and (3) to assess the relevance that the respondent assigns to a specific issue, including values like Not at all important, Not too important, Not sure /Not applicable, Somewhat important, and Very important.

In particular, Section A refers to the respondent age, country, language, background and the different FLOSS projects he/she have been enrolled in.

Section B explores the respondent's participation in a specific FLOSS project. In this section the respondent presents a specific project, to which he/she has (or is) contributing. The respondent is requested to describe how the participation started, the drivers what drove him to starting such activity, his/her role in the project, and how many hours he/she devotes to the project. The respondent has the opportunity to describe the type of relationships he has with community members, how they share information, or if they promote and have community meetings or events.

Section $\mathrm{C}$ aims at exploiting the potential of FLOSS projects as learning environments. In this section, the respondent analyzes whether the fact of being in a FLOSS community provides him with a learning opportunity, and if his background (professional or academic) facilitates the learning process while participating in a FLOSS project. We also explore who and what were the most important agents in his learning process, if FLOSS projects can be regarded as learning communities, if FLOSS can be seen as a possible alternative to formal education, and if FLOSS could be seen as an interesting complement to formal education.

\subsection{Questionnaire Construction and Dissemination Process}

The aim of the questionnaire was to (1) study the interactions between FLOSS projects participants, and (2) assess the didactical value of their communities. By interactions we mean the interactions between community members and the project, but also what drives an individual to start participating in FLOSS projects.

The questionnaire was carried out online using Google Docs Survey ${ }^{2}$. Following the same procedure as in [11], we tested the questionnaire, and performed a brief

\footnotetext{
${ }^{2}$ https://docs.google.com/a/iist.unu.edu/spreadsheet/ccc?key=0Akke8MV3ZtZidFpxdDRncTI3ekdj SIJzenFqNWVXNWc
} 
analysis, representing the first phase of activities. For the second phase, and before launching the questionnaire to broader audience, we carried out a pilot test. First, the questionnaire was re-introduced to the same FLOSS projects contributors of the first phase. This pilot test lasted for two weeks in May 2013. With the received feedback, the questionnaire was revised and improved. As part of the dissemination process, we contacted several institutions from associations (Drupal Association, Associação Portuguesa de Programadores Perl), and foundations (Perl Foundation) to companies (Google, RedHat, Citrix, Linux, OpenSource.com) through their Websites, Facebook, or Twitter pages.

Finally, the questionnaire was officially launched on 1 June 2013 and preliminary results collected by 13 June 2013. After collecting this first data, the questionnaire will remain available, in order to increase the sample of responses.

\section{Results}

Two weeks after the questionnaire was released, we were able to collect data from 27 respondents, from 16 different countries, including Portugal, United Kingdom, Germany, India, France, Serbia, Finland, Netherlands, Belgium, Slovenia, USA, Macau SAR China, Canada, Argentina, Israel, and Brazil. From the respondents, 24 are men and 3 are women. Respondents have different academic levels. The majority has postgraduate studies (41\%), followed by undergraduate studies (33\%). As far as their professional activities are concerned, $29.6 \%$ of the respondents are software developers, $18.5 \%$ students $-\mathrm{PhD}$ and undergraduate, and $3.7 \%$ are researchers. Concerning the list of projects, all respondents except one indicated only one project. The list of projects to which they contributed include: Perl, Perl Dancer, NetBSD, Gentoo Linux, Ubuntu Studio, Drupal, Kalkun, The Xen Project, and Joogie. A respondent who did not write any project name stated that he is involved in too many projects.

Results obtained in Section B are explained as follows. In Section A the respondents provided a list of projects. When asked to select a specific FLOSS project, $85 \%$ of the respondents presented different projects, although within a similar field of activity. $48 \%$ of the respondents work (or worked) on a project in the application software area, whereas $37 \%$ at the level of operating systems. Although $70 \%$ of the respondents participate in the correspondent project for more than 1 year, $19 \%$ indicate their participation in the project lasts for less than 6 months, as shown in Fig. 1.

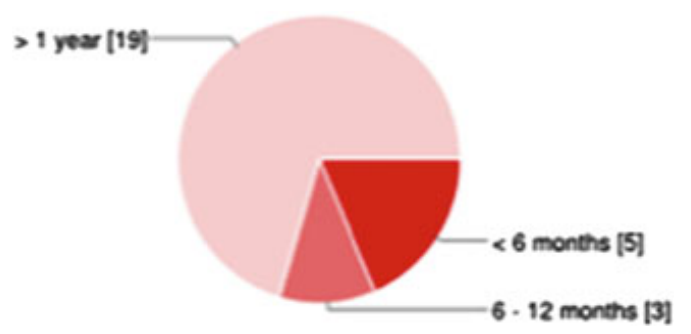

Fig. 1. Active participation in FLOSS projects 
Although for $56 \%$ of the respondents the challenge was what made them start participating in FLOSS projects, $52 \%$ answered that it was the idea that triggered their participation, as depicted in Fig. 2.

The activities that one can perform in a FLOSS project are several and may go from observer to developer, from user supporter to tester. Although $41 \%$ of the respondents stated that they started their participation as developers, it is important to notice that $44 \%$ of the respondents declared they started in other ways (a way not included among the available responses), as depicted in Fig. 3.

Among others, the respondents said that they started in the selected project as owner, or translator. Also, we determined that respondents started their participation by having more than one role, such as, tester and developer, or as developer and observer. Although respondents said they started with a certain role, it is interesting to analyze that $70 \%$ became active developers.

As far as the relationships are concerned, $33 \%$ of the respondents state that they are friends with other group members, $33 \%$ do not know any community members personally, $22 \%$ declared that the relationship is professional, and $11 \%$ have other types of relationships with community members. Concerning how often they collaborate with other group members, $56 \%$ said that more than 3 times per month, and $15 \%$ say they collaborate at least 3 times per month.

The channels used to share information and allowing collaboration in the community are diverse. They span from Wikis to Forums, from Mailing Lists to Chats. As far as the Wikis are concerned, $22 \%$ said they use them more than 3 times per month, whereas $22 \%$ said they never used them. As far as the Forums are concerned, $48 \%$

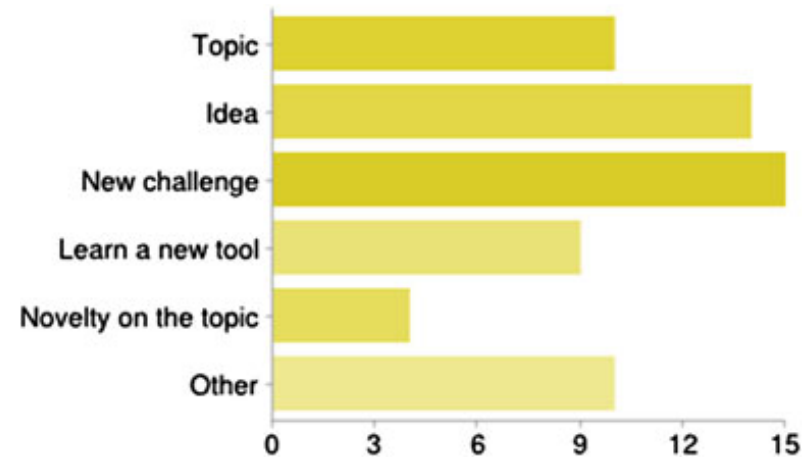

Fig. 2. Motivation to start contributing

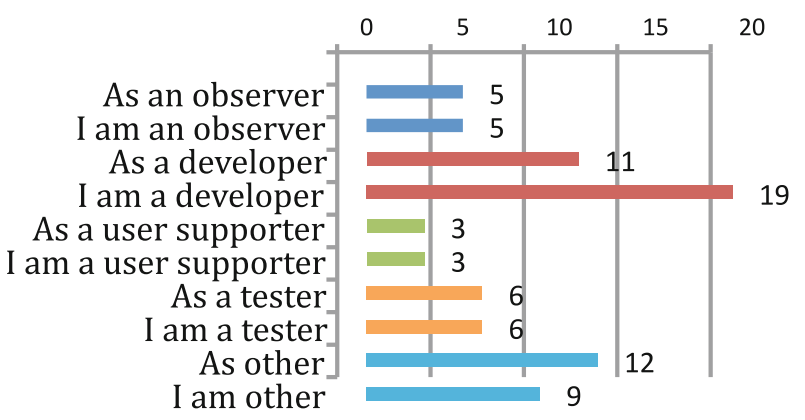

Fig. 3. Role at the beginning - As an...; and later - I am... 
said they never use this type of channel whereas $22 \%$ said they use them more than 3 times per month. As far as the Mailing lists are concerned, $44 \%$ of the respondents use it whereas $22 \%$ never use it. The channel with more usage is actually Chats where $63 \%$ of the respondents said they use it more than 3 times per month. Overall, $96 \%$ of the respondents use some sort of communication channel, whereas $4 \%$ don't communicate more than 3 times per month.

As far as meetings are concerned, $74 \%$ of the respondents have online meetings and $41 \%$ have face-to-face meetings. Concerning the type of events each community organize, $37 \%$ said conferences, $26 \%$ workshops, and $37 \%$ do not organize any types of events. Finally, $37 \%$ of the respondents believe that their participation in the projects can be improved, $26 \%$ strongly believes in that fact, whereas only $3 \%$ disagree, as depicted in Fig. 4.

The results obtained in Section $\mathrm{C}$ include the following. Several areas where FLOSS can provide expertise were included, such as testing, programming, code reviewing, code analysis, writing documentation, or reading and writing documentation.

As depicted in Fig. 5 respondents assessed their perception on the learning opportunities provided by selected activities as follows: (1) testing - $59 \%$ agree, (2) programming - $70 \%$ strongly agree, (3) code reviewing - $70 \%$ strongly agree, (4) writing documentation - $70 \%$ strongly agree, (5) reading and understanding documentation - $41 \%$ strongly agree. In addition, $89 \%$ strongly disagree that participating in a FLOSS project does not provide a learning opportunity, $7 \%$ only disagrees and $4 \%$ does not have an opinion about if participating in a FLOSS project can or not provide a learning opportunity.

If, as seen before, participating in a FLOSS project can be considered as a learning opportunity. It is also important to determine whether the background of the respondent - academic or professional - is a factor to the success of such learning experience. To this questions, $56 \%$ of the respondents agreed that their background is relevant for the success of their own learning process while participating in a FLOSS project, whereas $22 \%$ is not sure if it has an impact.

As far as the agents involved in their personal learning experience, $59 \%$ of the respondents agreed that themselves are very important in the learning process. $67 \%$ recognize that group members such as manager, senior members of the community or others, are important agents. $56 \%$ said that they are not sure if the end users are important agents, where $26 \%$ agrees that the end users are important agents in the learning experience.

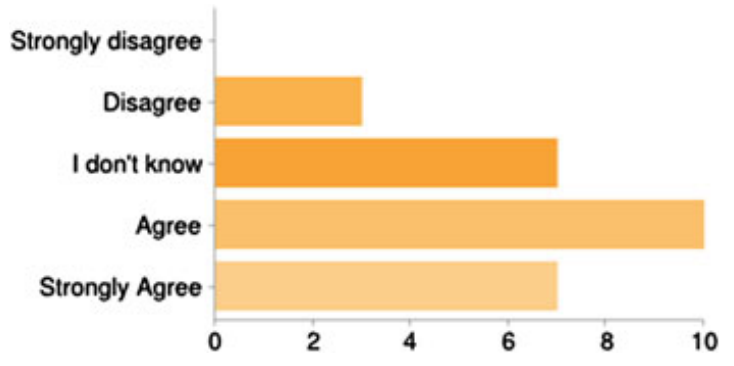

Fig. 4. Improvement through participation 


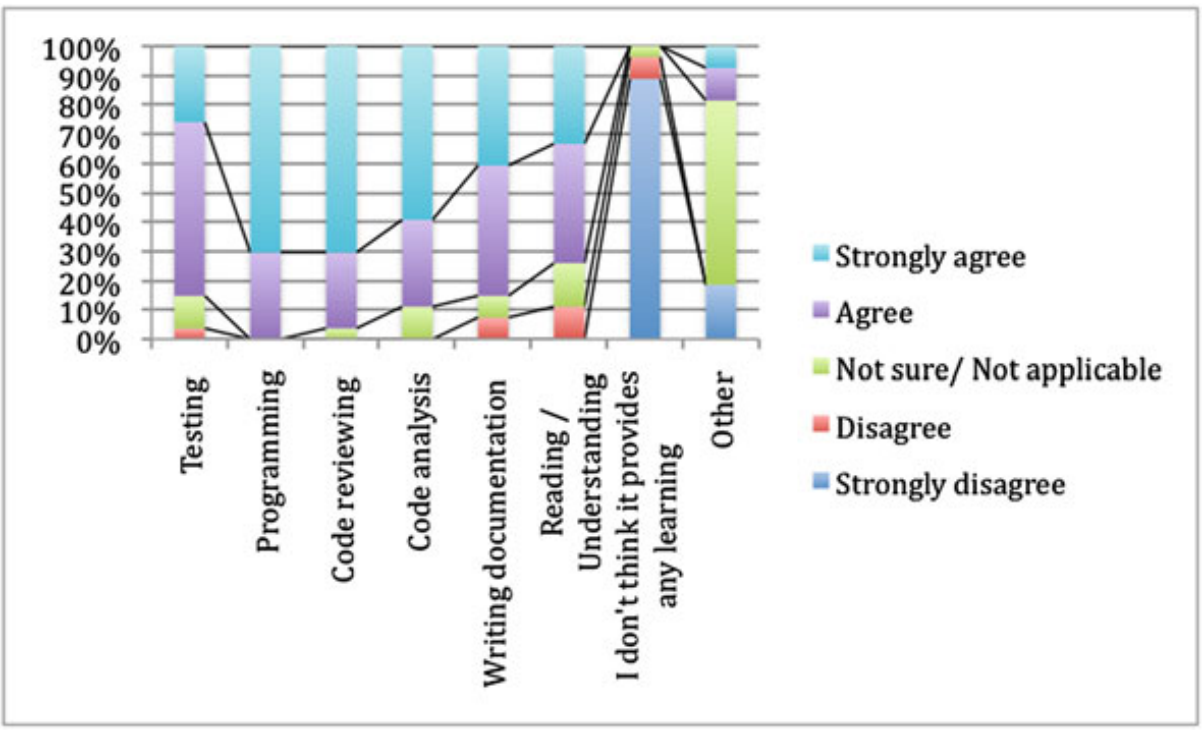

Fig. 5. FLOSS projects activities as learning opportunities

FLOSS projects can be regarded as an opportunity to perform several activities such as, attempting to understand shared code, programming, browsing and reading notes, documenting code, sharing concerns, contributing to the community forums, or reading formal publications such books, the answers. We have requested the respondents to evaluate such activities; responses are depicted in Fig. 6.

Respondents assess their perception on the relevance of conducting selected activities as follows: (1) attempting to understand shared code - $33 \%$ as very important and $19 \%$ as important, (2) programming - $44 \%$ as very important, (3) browsing and reading documentation - only $37 \%$ as somehow important, (4) documenting code - $30 \%$ as very important and $22 \%$ as somehow important, (5) sharing concerns, problems or solutions (using email, wikis, etc.) - $37 \%$ as very important,

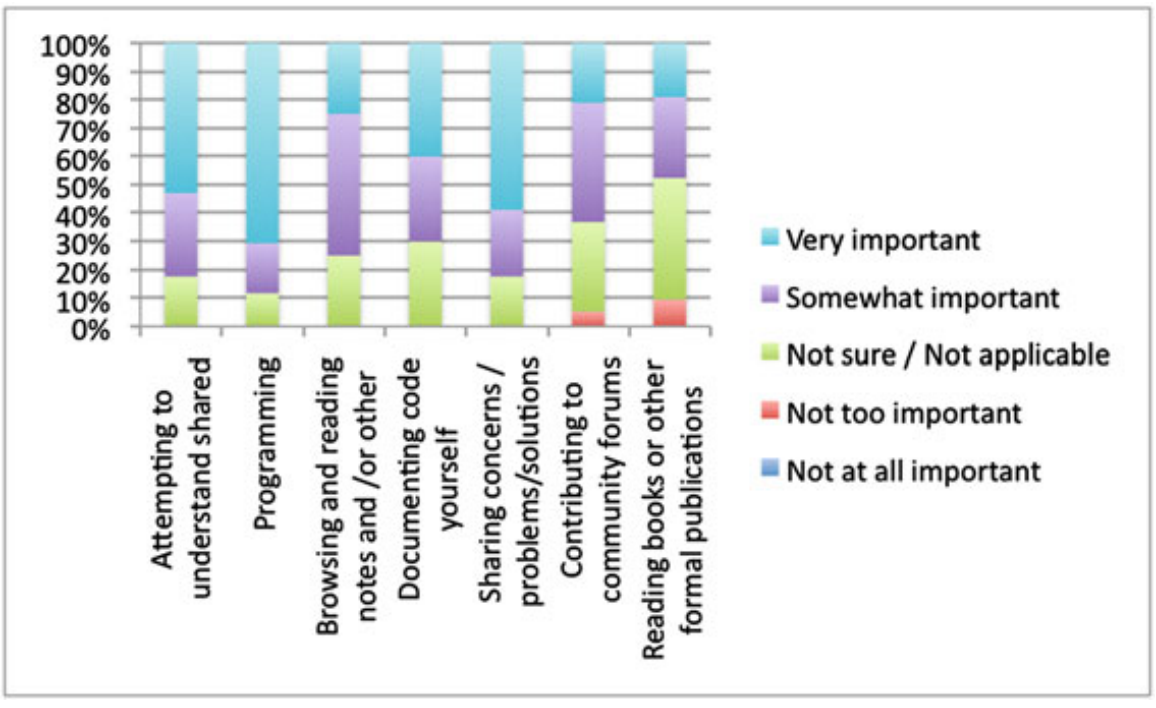

Fig. 6. Evaluation of different activities in FLOSS projects 
(6) contributing to community forums - $30 \%$ as somehow important, and (7) reading books or other formal publications $-33 \%$ are not sure.

When asked who were the most important agents in the respondents' learning experience, $59 \%$ of the respondents agree that themselves, and $41 \%$ strongly agrees with such fact. Also, $67 \%$ agrees that other group members are the most important agents in the respondents' learning experience, while only $26 \%$ strongly believe in this fact. However, and although the software developed in FLOSS communities are for general use, $56 \%$ do not see the End Users as agents of learning.

To the question, "has your involvement as a contributor in a FLOSS project changed the way you assess your own previous formal education", $37 \%$ agree that it changed whereas $26 \%$ are not sure.

In a more generic perspective, $48 \%$ of respondents agree that FLOSS projects communities can be regarded as learning communities, $41 \%$ strongly agree whereas only $4 \%$ disagree.

When questioned if FLOSS projects can be regarded as a possible alternative to formal education, $33 \%$ of the respondents do not agree. However, $59 \%$ strongly agree that it can be regarded as a complement to formal education. Results to this question are depicted in Fig. 7.

\section{Analysis}

For analyzing the results, we focus on 5 main dimensions:

(1) What - determining the type of interactions present (internal and external);

(2) How - determining how the respondents start their interactions, the modality they use to promote interactions, the impact of documentation available and the tools they use to interact;

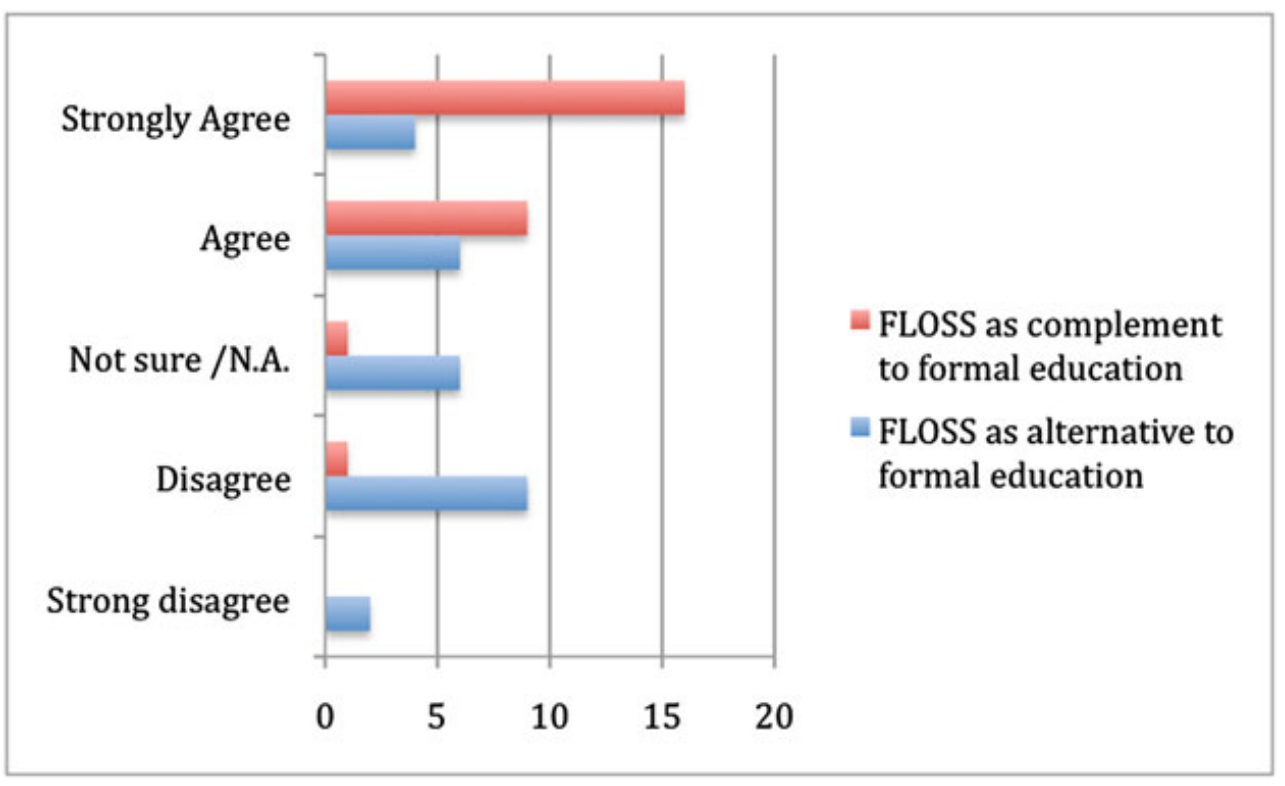

Fig. 7. Use of FLOSS projects in formal education 
(3) When - focusing on the frequency of interactions and contributions;

(4) Why - assessing their motivation to start the contribution on a FLOSS project, the impact of their background, the learning opportunities while participating in a FLOSS projects and the benefits of such participation; and

(5) Who - evaluating what roles the respondent play in the community and its learning agents.

The analysis for the above dimensions follows.

What - To determine the interactions within a FLOSS community, we first analyze what type of interactions exist: either they are internal interactions - where the respondent focuses on his personal experience with the community and the project or external, where the respondent presents how he promotes interaction with the community and to end-users of the product developed, such as events and meetings.

We have seen that nearly half of the respondents are friends with other community members. Nearly the remaining half simply does not know anybody in the community. Also, we have seen that nearly half of the respondents organize some sort of events. We believe that the high percentage of contributors that have a relationship, in this case, a friendly relationship, may have an impact on external interactions. The events organized, such as conferences and workshops, by default, promoting a direct contact among them.

How - In the questionnaire we presented many of the possible roles available in a FLOSS project. Interestingly, but not surprisingly, most of the respondents started their participation as developers and they continue being developers. They have online meetings and they use tools such as Wikis, Forums and Mailing lists to share information. Despite the intense use of such tools the majority of the respondents prefer chats to communicate. One of the main activities of the respondents is to develop software. As we know this is a type of work that is performed by single individuals. Such fact may indicate why contributors prefer chats to communicate. Besides chats, also mailing lists are very important to FLOSS contributors. Mailing lists are used as repositories by the community, as a way to share information, express concerns and achievements. Mailing lists, Wikis and Forums act as available documentation and are considered an asset for the project and the community.

When - From the results we determined that the majority of the respondents have been working in the selected project for more than 6 months. In average the respondents contribute to the project with more than $5 \mathrm{~h}$ per week, collaborating with other group members more than 3 times per month.

Why - The respondent background and initial motivations may have an impact on how committed he is to a project. It is interesting to see that most of the respondents said that they start participating in FLOSS projects as a challenge. Interestingly, 2 of the 3 women that replied to the questionnaire pointed that what made them start participation in FLOSS projects was the idea. As we know, the outcome of a FLOSS project is software. Not surprisingly the majority of the respondents have a background in computer science and are software developers. 
Who - The respondents' background, academic or professional, may explain why they started their participation as developers and they continue doing so. However, having the same role does not mean they don't acquire knew knowledge. When asked who are the most important agents of their learning experience, the respondents indicate themselves and the community. To them, not only the community can be regarded as a way to improve the learning process but also participation in FLOSS projects can be seen as a complement to formal education, where students can learn by doing, representing a positive impact on the learner.

Summary - Addressing the two research questions formulated in the Introduction: (1) study the interactions between FLOSS projects participants, and (2) assess the didactical value of their communities, our analysis shows the following conclusions.

\section{Conclusion and Future Work}

The aim of this paper was to (1) study the interactions of FLOSS community members, and (2) to assess the didactical value of such participation. As we were able to analyze by our small sample of respondents, FLOSS projects participants collaborate and cooperate between them. This let us with the certainty that there are interactions in FLOSS community members, and they are driven by the same goal: to develop a software project. It is important to verify that FLOSS community members, do not believe that the participation in FLOSS projects can be an alternative to formal education, for example, to replace formal courses in software engineering in higher education institutions. However, they see that the "learning by doing" concept can be applied in FLOSS projects and therefore is an attractive complement to formal education, mainly in software engineering courses. As future work, we aim comparing the data collected with a pilot project that uses FLOSS projects as assignments in Software Engineering courses.

Acknowledgments. This work is funded partly by UNU-IIST and Macau Foundation in the context of the PPAeL project. This work is funded by ERDF - European Regional Development Fund through the COMPETE Programme (operational programme for competitiveness) and by National Funds through the FCT - the Portuguese Foundation for Science and Technology, within project FCOMP-01-0124-FEDER-010049.

\section{References}

1. Anderson, P.: What is Web 2.0? Ideas, technologies and implications for education. http:// www.jisc.ac.uk/media/documents/techwatch/tsw0701b.pdf (2007). Accessed 11 Nov 2012

2. Fountain, R.: Wiki pedagogy. Dossiers Pratiques. Profetic. http://www.profetic.org:16080/ dossiers/dossier_imprimer.php3?id_rubrique=110 (2005). Accessed 11 Nov 2012

3. Chatti, M.A., Jarke, M., Frosch-Wilke, D.: The future of e-learning: a shift to knowledge networking and social software. Int. J. Knowl. Learn. 3(4/5), 404-420 (2007)

4. Downes, S.: Web 2.0 and your own learning and development. http://www.elearnspace.org/ blog/2007/06/19/web-20-and-your-own-learning-and-development/ (2007). Accessed 10 May 2013 
5. Happ, S.: The changing world of e-learning. http://besser20.de/the-changingworld-of-e-learning/44/ (2008). Accessed Dec 2012

6. Sclater, N.: Web 2.0, personal learning environments and the future of learning management systems. Educause Res. Bull. (13 Boulder, CO: Educause Center for Applied Research) (2008)

7. Wilson, S., Liber, P.O., Johnson, M., Beauvoir, P., Sharples, P.: Personal learning environments: challenging the dominant design of educational systems. J. e-Learn. Knowl. Soc. 3(2), 27-38 (2007)

8. Downes, S.: Knowledge, learning and community. http://www.downes.ca/post/57737 (2012). Accessed 10 Nov 2012

9. Fernandes, S., Cerone, A., Barbosa, L.S.: FLOSS communities as learning networks. Int. J. Inf. Educ. Technol. 3(2), 278-281 (2013)

10. Cerone, A., Sowe, S.K.: Using free/libre open source software projects as learning tools. In: OpenCert 2010, vol. 33 of ECEASST (2010)

11. Fernandes, S., Cerone, A., Barbosa, L.S.: A preliminary analysis of learning awareness in FLOSS projects. In: International Symposium on Innovation and Sustainability in Education (INSUEDU 2012). Springer, Thessaloniki (2012, in Press)

12. Times Magazine: http://www.time.com/time/covers/0,16641,20061225,00.html (2013). Accessed 5 May 2013

13. Brocco, A., Frapolli, F.: Open Source in Higher Education: Case Study Computer Science at the University of Fribourg (2011)

14. Papadopoulos, P.M., Stamelos, I.G., Meiszner, A.: Students' perspectives on learning software engineering with open source projects: lessons learnt after three years of program operation. In: Proceedings of the Fourth International Conference on Computer Supported Education (CSEDU 2012), pp. 313-322 (2012)

15. Magrassi, P.: Free and open-source software is not an emerging property but rather the result of studied design. In: ICICKM10 (2010)

16. Lundell, B., Persson, A., Lings, B.: Learning through practical involvement in the FLOSS ecosystem: experiences from a master assignment. In: Proceedings of the Third International Conference on Open Source Systems 2007, pp. 289-294 (2007) 